\title{
Electronic and vibronic properties of a discotic liquid-crystal and its charge transfer complex
}

\author{
Lucas A. Haverkate, ${ }^{1}$ Mohamed Zbiri, ${ }^{2, a)}$ Mark R. Johnson, ${ }^{2}$ Elizabeth Carter, ${ }^{3}$ Arek \\ Kotlewski, ${ }^{4}$ S. Picken, ${ }^{4}$ Fokko M. Mulder, ${ }^{1}$ and Gordon J. Kearley ${ }^{5}$ \\ ${ }^{1}$ Reactor Institute Delft, Faculty of Applied Sciences, Delft University of Technology, Mekelweg 15, \\ 2629JB Delft, The Netherlands \\ ${ }^{2}$ Institut Laue Langevin, 38042 Grenoble Cedex 9, France \\ ${ }^{3}$ Vibrational Spectroscopy Facility, School of Chemistry, The University of Sydney, \\ NSW 2008, Australia \\ ${ }^{4}$ ChemE-NSM, Faculty of Chemistry, Delft University of Technology, 2628BL/136 Delft, The Netherlands \\ ${ }^{5}$ Bragg Institute, Australian Nuclear Science and Technology Organisation, Menai, NSW 2234, Australia
}

(Received 10 April 2013; accepted 11 December 2013; published online 6 January 2014)

\begin{abstract}
Discotic liquid crystalline (DLC) charge transfer (CT) complexes combine visible light absorption and rapid charge transfer characteristics, being favorable properties for photovoltaic (PV) applications. We present a detailed study of the electronic and vibrational properties of the prototypic 1:1 mixture of discotic 2,3,6,7,10,11-hexakishexyloxytriphenylene (HAT6) and 2,4,7-trinitro9-fluorenone (TNF). It is shown that intermolecular charge transfer occurs in the ground state of the complex: a charge delocalization of about $10^{-2}$ electron from the HAT6 core to TNF is deduced from both Raman and our previous NMR measurements [L. A. Haverkate, M. Zbiri, M. R. Johnson, B. Deme, H. J. M. de Groot, F. Lefeber, A. Kotlewski, S. J. Picken, F. M. Mulder, and G. J. Kearley, J. Phys. Chem. B 116, 13098 (2012)], implying the presence of permanent dipoles at the donor-acceptor interface. A combined analysis of density functional theory calculations, resonant Raman and UV-VIS absorption measurements indicate that fast relaxation occurs in the UV region due to intramolecular vibronic coupling of HAT6 quinoidal modes with lower lying electronic states. Relatively slower relaxation in the visible region the excited CT-band of the complex is also indicated, which likely involves motions of the TNF nitro groups. The fast quinoidal relaxation process in the hot UV band of HAT6 relates to pseudo-Jahn-Teller interactions in a single benzene unit, suggesting that the underlying vibronic coupling mechanism can be generic for polyaromatic hydrocarbons. Both the presence of ground state CT dipoles and relatively slow relaxation processes in the excited CT band can be relevant concerning the design of DLC based organic PV systems.

(C) 2014 AIP Publishing LLC. [http://dx.doi.org/10.1063/1.4856815]
\end{abstract}

\section{INTRODUCTION}

Discotic liquid crystals (DLCs) are considered as a promising class of organic materials for photovoltaic (PV) and other electronic applications. ${ }^{1-7}$ These disk-like molecules form stable columns due to the $\pi-\pi$ orbital overlap of their aromatic cores, while thermal fluctuations of their side chains give rise to the liquid-like dynamic disorder. ${ }^{8}$ DLCs combine advantageous materials properties, including visible light absorption, long-range self-assembly, selfhealing mechanisms, high charge-carrier mobilities along the column axis, and a tunable alignment of the columns. Like conjugated polymers, DLCs offer the potential of low-cost, easily processed and flexible solar cells. ${ }^{4,9}$ But, a major challenge to application is to achieve a morphology that enables a bulk-heterojunction (BHJ) PV device architecture. $^{6,10}$ This topic has attracted considerable interest over the past decade, with the goal of obtaining an interpenetrating network of electron and hole conducting

a) Author to whom correspondence should be addressed. Electronic mail: zbiri@ill.fr molecular columnar wires enabling donor-acceptor (D-A) phase separation on the nanoscale. ${ }^{1,3,11,12}$

Another crucial issue for organic PV application is the strong electron-phonon coupling inherent to molecular systems, which limits the efficiency of charge separation. ${ }^{10}$ Upon photoexcitation, strongly bound exciton states are formed that first need to dissociate before charge transport to the electrodes can occur. Dissociation takes place at the D-A interface, where intermediate excited charge-transfer (CT) states are formed with the hole on the donor and electron at the acceptor molecule. At present time there is no clear picture of the final charge separation process, i.e., the dissociation of the excited CT state into a free electron and hole. The lowest CT state corresponds to a Coulombically bound electron-hole excited pair, with a binding energy of typically several hundreds of meV. ${ }^{13}$ Various scenarios have been proposed to explain separation of charges from this lowest state, including the possible presence of dipoles at the D-A interface. ${ }^{10,14}$ Other studies indicate that charge separation is mediated by the higher lying vibronic states of the excited CT manifold. ${ }^{15,16}$ In such a process, charge carriers undergo a few ultrafast hops via an activationless pathway, allowing their separation before 
the thermal relaxation and towards the occurrence of localized excitonic levels. In this respect, fundamental knowledge of the electronic and vibrational properties of the excited state levels and relaxation pathways is a key topic in further understanding and improving the working principles of OPVs. ${ }^{15}$

This paper investigates the possible presence of permanent dipoles at the D-A interface of HAT6-TNF compound. Further, a first step is presented to characterize the influence of molecular vibrations on the charge carrier relaxation in self-assembled DLCs. For self-assembled aggregates, such as DLCs and DLC ground state CT complexes, the characterization of photo-induced electron transfer and relaxation processes is at an early stage. ${ }^{17}$ The addition of electron acceptors has been shown to increase the conductivity of DLCs. ${ }^{18-20}$ On the other hand, it has been proposed that recombination processes limit the hole photocurrent in such compounds. ${ }^{21}$ Charge carriers in ground state CT compounds are supposed to be trapped and readily annihilated through rapid, phononassisted relaxation and recombination processes. ${ }^{3,21,22}$

The prototypical discotic electron donating discoid HAT6 and its 1:1 mixture with electron acceptor TNF (Fig. 1) is chosen as a model system. HAT6-TNF forms a ground state CT compound exhibiting a stable columnar phase from below room temperature to $237^{\circ} \mathrm{C} . .^{23}$ The high symmetry and moderate molecular size of discogens such as HAT6 makes these systems attractive for exploring the effects of increasing molecular complexity, by comparing their photophysical properties with those of the fundamental building block, benzene, and large polyaromatic hydrocarbons. ${ }^{24-26}$

We start with an investigation of the electronic properties of HAT6-TNF. For discotic liquid crystalline complexes, formed by donor and acceptor molecules, it is generally accepted that the intermolecular charge transfer is of an excited state nature. ${ }^{27,28}$ Indeed, mixtures of the electron donating discoids with non-discogenic electron acceptors exhibit absorption bands in the visible region due to excited state charge transfer. ${ }^{29-31}$ However, recently we found indications of weak electron transfer occurring in the ground-state of HAT6-TNF. ${ }^{32}$ This implies the presence of permanent dipoles between the donor (HAT6) and acceptor (TNF) molecules. Here, we find strong support for these indications from a com-

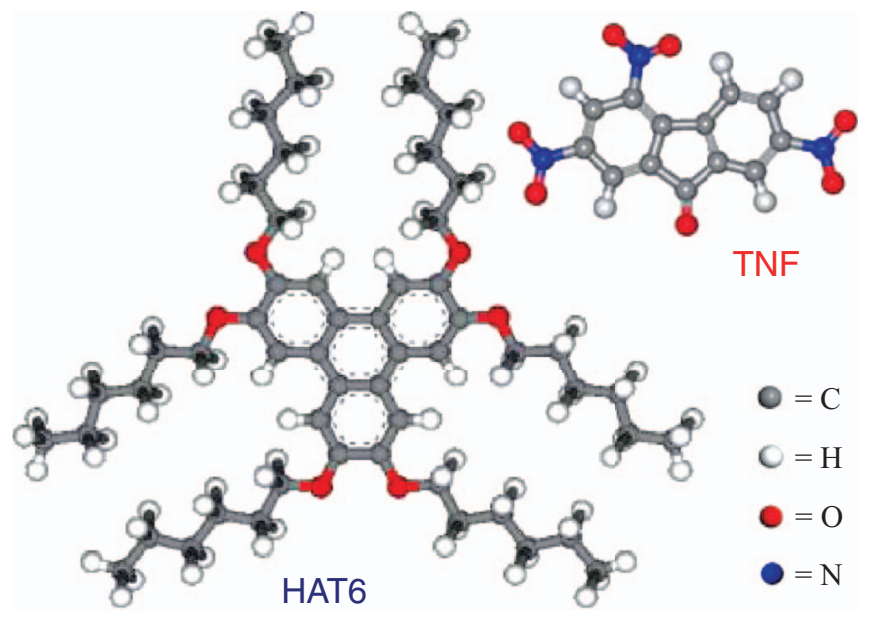

FIG. 1. Illustration of HAT6 (hexakis(n-hexyloxy)triphenylene) and TNF (2,4,7-trinitro-9-fluorenone) molecules. bination of our previous NMR results ${ }^{32}$ with the present Raman spectroscopy measurements. In addition, we characterize the electronic transitions involved in the excited CT-band of HAT6-TNF by combining UV-VIS absorption and resonant Raman spectroscopy. Subsequently, the UV-VIS and Raman measurements are combined with density functional theory (DFT) calculations, to identify the vibrational modes that assist charge-carrier relaxation in the "hot" band of HAT6 and in the excited CT-band of HAT6-TNF.

\section{MATERIALS AND METHODS}

\section{A. Sample preparation}

Isotopically normal 2,3,6,7,10,11-hexakishexyloxytriphenylene (HAT6) and its side-chain deuterated analog, HAT6D, were prepared by the synthesis methods described earlier. ${ }^{23,33}$ The charge transfer compounds were obtained by mixing HAT6 (or HAT6D) with 2,4,7-trinitro-9-fluorenone (TNF) in a 1:1 molar proportion in dichloromethane. ${ }^{23}$ The mixture was subsequently evaporated to dryness at room temperature. To remove any traces of solvent and to ensure the correct phase behavior, the resulting composite was heated to the isotropization temperature, $\mathrm{T}=237^{\circ} \mathrm{C}$, then cooled slowly. By using a deuterated analog for TNF as well (TNFd, with all hydrogens deuterated), four different analogues were obtained: HAT6-TNF, HAT6D-TNF, HAT6-TNFd, and HAT6D-TNFd. The degree of deuteration of HAT6D and TNFd was about 98 atom\%.

\section{B. Absorption spectroscopy}

Optical absorption at room temperature was measured using a Perkin-Elmer Lambda 900 spectrometer equipped with an integrating sphere. The optical density was measured and the attenuation $F$ a (fraction of incident photons that is absorbed by the sample) was obtained by correction for reflection losses.

\section{Raman spectroscopy}

Spectra at wavelengths of $1064,785,633,514$, and $488 \mathrm{~nm}$ were collected at the Vibrational Spectroscopy Facility (School of Chemistry, The University of Sydney (USYD)). The off-resonance spectra at $1064 \mathrm{~nm}$ were recorded with a Bruker FT-Raman (MulitRAM) spectrometer using a $\times 100 / 1.25$ NA objective, with the laser power at the sample spot between 50 and $200 \mathrm{~mW}$ depending on the sample. The spectra at 785, 633, 514, and $488 \mathrm{~nm}$ were obtained with a Renishaw Raman InVia Reflex Microscope (Renishaw plc., Wotton-under-Edge, UK), using a $\times 50 / 0.75$ NA objective and a laser power of $0.1-1.0 \mathrm{~mW}$. The details of this spectrometer have been described in Ref. 34. A Renishaw Raman InVia Reflex Microscope (Renishaw plc., Wotton-under-Edge, UK) spectrometer located at the Analytical Centre, The University of New South Wales (UNSW) was used to collect spectra at an excitation wavelength of $325 \mathrm{~nm}$, the samples being measured with a $\times 40$ objective and the laser power at the sample being estimated as between 0.8 and $1.0 \mathrm{~mW}$. Spectra were obtained from different positions on a selected sample region, the 
number of selected spots varying between 10 and 50 depending on the sample and wavelength, these spectra were then averaged. The accumulation and exposure times were typically 10-50 and $10 \mathrm{~s}$, respectively.

\section{Raman simulations}

Raman scattering activities were simulated adopting the Kohn-Sham formulation of the density functional theory $(\text { DFT) })^{35,36}$ as implemented in the Gaussian 03 program (version D.01). ${ }^{37}$ The input HAT6 geometry (144 atoms) was built by considering structures of benzene and hexalkoxygroups for the aromatic core and tails $\left(\mathrm{R}=\mathrm{OC}_{6} \mathrm{H}_{13}\right)$, respectively. An initial fully planar $\mathrm{D}_{3 \mathrm{~h}}$ symmetry was assumed, the only out-of-plane atoms being the hydrogens of the tails. All calculations were performed on a Beowulf Intel cluster at the Delft University of Technology (the Netherlands). Several combinations of exchange-correlation (XC) functionals (local, semilocal, and nonlocal) and basis sets (including or not polarization and diffuse functions) have been tested to establish the best model calculation(s) that lead to an explanation of experimental observations. The technicalities and methodologies used in the calculations are beyond the scope of the current topic and will not be discussed here. The predicted Raman activities were simulated in the gas phase adopting the SVWN XC functional, which consists of the Slater exchange $(\mathrm{S})^{38}$ combined to the Vosko, Wilk, and Nusair approxima- tion (VWN) of the correlation part. ${ }^{39}$ Pople's group basis set 6-311G** was adopted for all atomic types. ${ }^{40}$ A Gaussian function of a FWHM of $10 \mathrm{~cm}^{-1}$ was convoluted with the calculated Raman data to take account the resolution in the measured spectra.

\section{RESULTS}

\section{A. Absorption}

It is well established that discotic compounds change color upon complexation with an electron acceptor. ${ }^{3,29}$ For HAT6-TNF this color change is quite strong: the 1:1 LC mixture of the white colored HAT6 and the yellow electron acceptor TNF becomes black. Indeed, the absorption spectrum of HAT6-TNF (Fig. 2(a)) shows a broad excited CT-band extending from $\sim 500 \mathrm{~nm}$ to about $870 \mathrm{~nm}$, the band gap thus being below $1.43 \mathrm{eV}$. In contrast, HAT6 shows a strong absorption band at $366 \mathrm{~nm}$ and only weakly allowed transitions at longer wavelengths. Triphenylene and HAT6 absorption spectra have already been studied extensively in literature. ${ }^{41-43}$ Based on the similarity with the present liquid crystalline measurement, we assigned the small absorption peaks at 469 and $442 \mathrm{~nm}$ to the formally forbidden $\mathrm{S}_{0} \rightarrow \mathrm{S}_{1}\left(\mathrm{~A}_{1}^{\prime} \rightarrow \mathrm{A}^{\prime}{ }_{1}\right)$ electronic transition, a shoulder around $417 \mathrm{~nm}$ to $S_{0} \rightarrow S_{2}$ $\left(\mathrm{A}_{1}^{\prime} \rightarrow \mathrm{A}_{2}^{\prime}\right)$, the broad peak at $402 \mathrm{~nm}$ to $\mathrm{S}_{3}\left(\mathrm{~A}_{1}^{\prime} \rightarrow \mathrm{E}^{\prime}\right)$ and the absorption maximum at $366 \mathrm{~nm}$ to the strong allowed $\mathrm{S}_{4}$

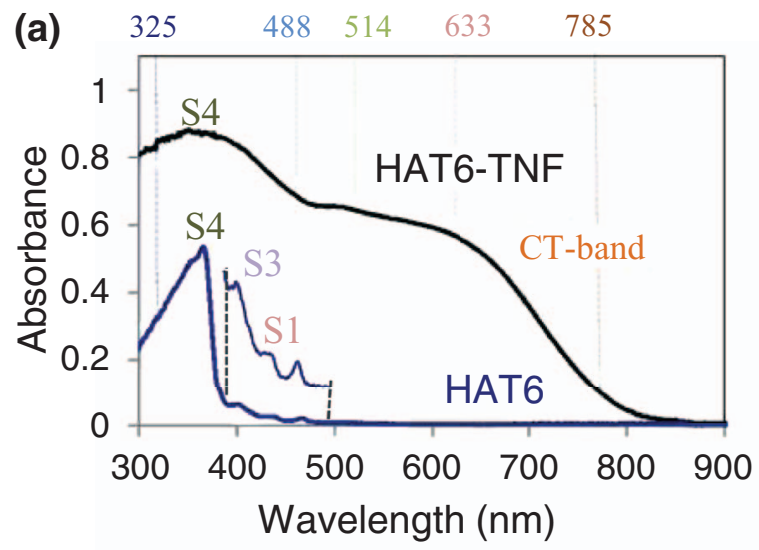

(b)

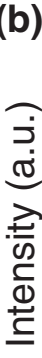
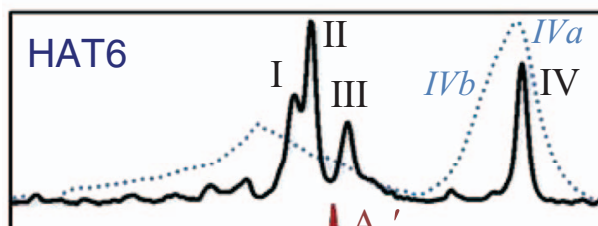

... $325 \mathrm{~nm}$

$$
\begin{array}{r}
-1064 \mathrm{~nm} \\
-\quad \text { simulated }
\end{array}
$$

1100

1300

Wavenumber $\left(\mathrm{cm}^{-1}\right)$

(c)

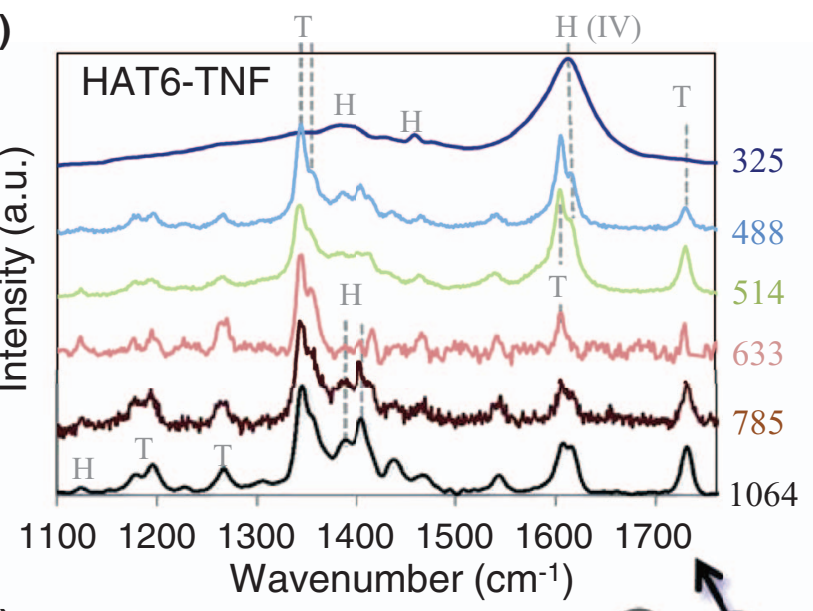

(d)

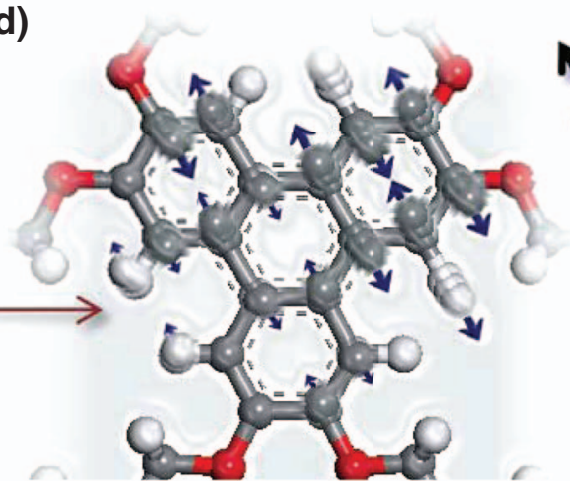

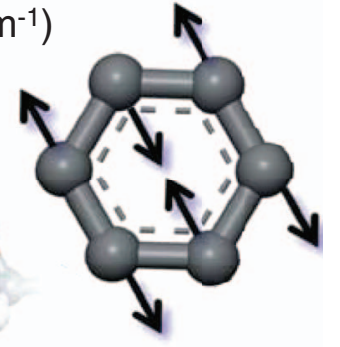

$\mathrm{E}_{2 \mathrm{~g}}$ mode

benzene

FIG. 2. Overview of the spectroscopic results. (a) Absorption spectra of HAT6 at $350 \mathrm{~K}$ and HAT6-TNF at $300 \mathrm{~K}$, including the assignment of the absorption bands. (b) (Top) Off-resonance (1064 nm, solid line) and resonance (325 nm, dotted line) Raman spectra for HAT6 at $300 \mathrm{~K}$. (Bottom) Off-resonance Raman activities profile calculated with DFT. (c) Raman spectra for HAT6-TNF at $300 \mathrm{~K}$ for the indicated wavelengths. The assignment of the peaks is labeled as H for HAT6 and T for TNF. (d) Illustration of the $\mathrm{E}^{\prime}$ normal mode calculated at $1628 \mathrm{~cm}^{-1}$ (left) and its similarity with the quinoidal E $2 \mathrm{~g}$ mode of benzene (right). 
$\left(\mathrm{A}^{\prime}{ }_{1} \rightarrow \mathrm{E}^{\prime}\right)$ transition. The $\mathrm{S}_{4}$ transition of HAT6 also dominates the higher energy region in the excited CT complex. The electronic absorption spectrum of TNF has been studied in Refs. 44 and 45 and contains a weak lowest-energy band at a wavelength of $435 \mathrm{~nm}$ due to the $\mathrm{n}-\pi *$ transition and higher energy bands at $387,302,260$, and $222 \mathrm{~nm}$ due to $\pi-\pi^{*}$ transitions. Thus, the 500-870 $\mathrm{nm}$ region where the excited CTband is situated is clear of any electronic transitions from pure HAT6 or TNF. The CT band is a result of the excited chargetransfer interaction between HAT6 and TNF involving electronic transition from the highest occupied molecular orbitals (HOMOs) of HAT6 to the lowest unoccupied molecular orbitals (LUMOs) of TNF, although the molecular orbitals become mixed in the excited CT complex. ${ }^{31(a), 31(b)}$

\section{B. Raman spectroscopy}

A complete overview of the off-resonance Raman spectra for HAT6, TNF, and HAT6-TNF is presented in the supplementary material. ${ }^{46}$ We assigned TNF by using DFT calculations in combination with analyses presented in earlier studies. ${ }^{44,47,48}$ The most relevant Raman modes (Table S3) are due to symmetric $\mathrm{C}-\mathrm{NO}_{2}$ stretching $\left(\sim 1350 \mathrm{~cm}^{-1}\right)$, $\mathrm{C}-\mathrm{C}$ skeleton vibration $\left(1601 \mathrm{~cm}^{-1}\right)$ and $\mathrm{C}=\mathrm{O}$ stretching $\left(1733 \mathrm{~cm}^{-1}\right)$. There is good agreement between the measured and simulated Raman data (Fig. 2(b)) for HAT6, which enabled us to assign most of the bands. Of prime importance is the $\mathrm{E}^{\prime}$ symmetric vibration IV at $1617 \mathrm{~cm}^{-1}$, which is situated at the aromatic core of HAT6. It bears a strong resemblance to the quinoidal $v_{8}\left(E_{2 \mathrm{~g}}\right)$ mode of benzene ${ }^{24,25,49}$ as illustrated in Fig. 2(d) and Fig. S2.

The off-resonant Raman spectrum of the ground state CTcomplex is a superposition of the HAT6 and TNF vibrational modes, which were assigned by comparison with the spectra of the uncomplexed components. ${ }^{46}$ However, several vibrational modes are considerably shifted in frequency, and it is well established that these shifts of donor and acceptor frequencies are related to the occurrence of a ground state charge transfer. ${ }^{4,50-54}$ In particular, for the acceptor, TNF, a redshift of the $\mathrm{C}=\mathrm{O}$ stretching mode has been observed in ground state $\mathrm{CT}$ complexes and is related to an increase in the electron density resulting from partial ground-state $\mathrm{CT} .{ }^{48,52} \mathrm{In}$ the HAT6-TNF spectrum, the $\mathrm{C}=\mathrm{O}$ mode is observed to have redshifted to $1730 \mathrm{~cm}^{-1}$. Based on the data in literature ${ }^{52}$ it is estimated that the shift of $3 \mathrm{~cm}^{-1}$ corresponds to an increase in electron density on TNF of about $0.03 \mathrm{e}^{-}$. In addition, for all the assigned modes we found satisfactory agreement between the observed shifts and normalized frequency changes between neutral TNF and the anion $\mathrm{TNF}^{-1}$ calculated with DFT (Table S4), with the normalization factor corresponding to a ground state charge transfer of about 0.06 electron.

The electronic transitions in the excited CT complex and the vibrational modes involved in these transitions were investigated by exciting at different wavelengths in the absorption band (Fig. 2(a)). The corresponding resonant Raman spectra are shown in Fig. 2(c) $\left(1100-1800 \mathrm{~cm}^{-1}\right.$ region) and Fig. S6 (500-1000 $\left.\mathrm{cm}^{-1}\right){ }^{46}$ The resonance Raman spectra at 633 and $785 \mathrm{~nm}$ were obtained after subtraction of a broad luminescence background (Fig. S5), which results in a lower signal to noise ratio. The resonance Raman spectra are dominated by the TNF vibrational bands, especially the symmetric $\mathrm{C}-\mathrm{NO}_{2}$ stretching modes around $1350 \mathrm{~cm}^{-1}$ when exciting in the low energy region of the excited CT-band (785 and $633 \mathrm{~nm}$ ). Most of the HAT6 vibrational modes (e.g., I, II, III, and IV) are considerably less intense, or even absent, compared with the off-resonance case. However, the lowfrequency radial breathing mode of the HAT6 aromatic core at $721 \mathrm{~cm}^{-1}$ shows significant intensity for both excitation wavelengths (Fig. S6) ${ }^{46}$ The activity of the symmetric $\mathrm{C}-\mathrm{NO}_{2}$ vibrations and the radial breathing mode of the HAT6 core suggest that the lowest electronic transition in the excited CT complex is due to a charge transfer from the HAT6 core to TNF, with a strong involvement of the nitro groups. Strong resonant activity of symmetric $\mathrm{C}-\mathrm{NO}_{2}$ stretching modes is also observed for small aromatic nitro compounds with intramolecular ground state $\mathrm{CT}^{55}$ In these molecules the $\pi$ $\pi^{*}$ electronic transition of the lowest excited state gives rise to significant bond length and bond angle changes in the $\mathrm{C}-\mathrm{NO}_{2}$ group, reflecting that the excited state wavefunction contains a large contribution from the basis functions of the nitro group. ${ }^{55-59}$ For HAT6 it is well established that the HOMO is located on the aromatic core. ${ }^{60,61}$ Based on these considerations, we propose that the lowest excited state in HAT6-TNF forming the lower energy region of the excited CT-band is predominantly a $\pi-\pi^{*}$ type transition involving the highest occupied molecular orbitals (HOMO, HOMO-1, etc.) of HAT6 and the lowest unoccupied molecular orbitals from TNF (LUMO, LUMO+1, etc), ${ }^{31(b)}$ with a prominent role of the TNF nitro groups. Additional support for this assignment is obtained from DFT calculations on TNF, ${ }^{46}$ showing that the LUMO is of $\pi^{*}$-type and contains a predominant contribution from the nitro groups.

The resonant spectra, after exciting higher energy in the CT-band, are significantly different from the spectra at 785 and $633 \mathrm{~nm}$, although there is still significant activity of the symmetric $\mathrm{C}-\mathrm{NO}_{2}$ stretching modes for excitation at both 514 and $488 \mathrm{~nm}$. But the most strongly enhanced TNF mode, compared to off-resonance, is the $\mathrm{C}-\mathrm{C}$ skeleton vibration at $1604 \mathrm{~cm}^{-1}$, whilst for HAT6 there is a strong resonant activity of the quinoidal vibration IV. The broad excited CT band thus seems to consist of a superposition of at least two different electronic transitions, involving different molecular orbitals of HAT6 and TNF. Such a superposition agrees with the small shoulder observed in the excited CT-band around $510 \mathrm{~nm}$ (Fig. 2(a)) and partly explains the significant width of this band. However, from the similarity of the 785 and $633 \mathrm{~nm}$ spectra it also appears that the separate transitions give rise to rather broad absorption signals, which is consistent with the significant dynamic disorder in donor-acceptor distances found in the structural study. ${ }^{32}$

For excitation at $325 \mathrm{~nm}$ we can compare the CT-compound directly with the pure HAT6 and TNF compounds under resonance conditions. The resonant Raman spectrum of HAT6-TNF (Fig. 2(c)) compares well with that of pure HAT6 (Fig. 2(b)), both being significantly broadened and showing an envelope of bands with a maximum at about $1360 \mathrm{~cm}^{-1}$ for HAT6 and $1380 \mathrm{~cm}^{-1}$ for HAT6-TNF. The 

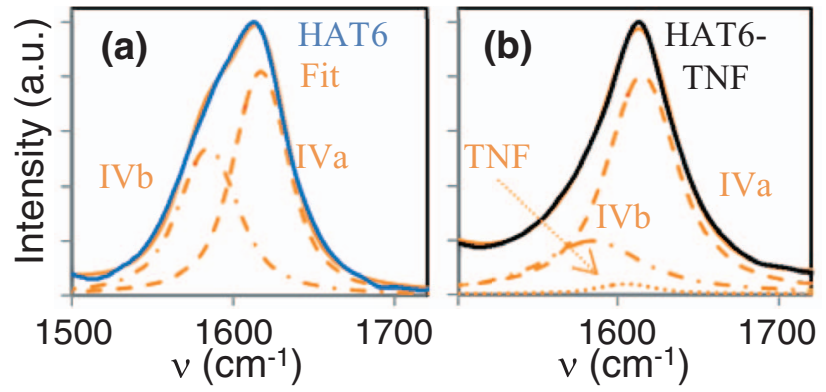

FIG. 3. Resonant Raman spectra of the $\mathrm{E}^{\prime}$ vibration IV for HAT6 (a) and HAT6-TNF (b) at $325 \mathrm{~nm}$, fitted with Voigt lineshapes (orange). The mode is split into a IVa (dashed) and a vibronic IVb (dashed-dotted) channel for both HAT6 and HAT6-TNF. The fitted contribution of the TNF band at $1606 \mathrm{~cm}^{-1}$ (dotted line) is very small.

$325 \mathrm{~nm}$ spectrum of TNF, on the other hand, looks rather different with considerably narrower lines (Fig. S7). ${ }^{46}$ These observations are all consistent with the proposition that the electronic transition relating to the high energy $(\sim 366 \mathrm{~nm})$ absorption band of HAT6-TNF strongly resembles the $\mathrm{S}_{4}$ $\left(A^{\prime}{ }_{1} \rightarrow E^{\prime}\right)$ transition of pure HAT6. The most enhanced mode in the $325 \mathrm{~nm}$ spectra of HAT6 and the excited CT-complex is the quinoidal $\mathrm{E}^{\prime}$ symmetric HAT6 mode at about $1616 \mathrm{~cm}^{-1}$. For pure HAT6 a shoulder is also clearly visible on this band, with a maximum considerably below $1600 \mathrm{~cm}^{-1}$. The offresonance spectrum of HAT6, on the other hand, only shows a weakly Raman-active mode at about $1592 \mathrm{~cm}^{-1}$, in addition to mode IV at $1617 \mathrm{~cm}^{-1}$. Similar observations exist for HAT6-TNF, although the shoulder is less clearly visible and a small contribution of the TNF C-C skeleton mode cannot be excluded. The $1500-1700 \mathrm{~cm}^{-1}$ region of both spectra were fitted with two Voigt lineshapes; denoted with IVa and IVb (Fig. 3 and Table I). An extra lineshape representing the TNF $\mathrm{C}-\mathrm{C}$ skeletal mode was included for HAT6-TNF, with a fixed frequency $1605 \mathrm{~cm}^{-1}$. The fitted peak positions of mode IVa are in good agreement with the observed frequencies for the $E^{\prime}$ symmetric vibration in the off-resonance spectra of HAT6 $\left(1617 \mathrm{~cm}^{-1}\right)$ and HAT6-TNF $\left(1615 \mathrm{~cm}^{-1}\right)$. The fitted intensity for the TNF C-C skeletal mode is very small $(\sim 1 \%$ of the total area), confirming that the $325 \mathrm{~nm}$ resonant enhancement of TNF is not significant in the excited CT-complex (see also Fig. S7). Most importantly, the fit shows that a strong contribution of a second mode IVb must be present in both spectra, with a frequency of about $1585 \mathrm{~cm}^{-1}\left(1584 \mathrm{~cm}^{-1}\right)$ for HAT6 (HAT6-TNF). The integrated intensity of the IVb is about $44 \%$ of the total peak area for pure HAT6, and about $24 \%$ for HAT6-TNF.

TABLE I. Results of the fit shown in Fig. 3.

\begin{tabular}{lccc}
\hline \hline Mode & Position $\left(\mathrm{cm}^{-1}\right)$ & FWHM $\left(\mathrm{cm}^{-1}\right)$ & Area (\%) \\
\hline IVa HAT6 & $1616.8 \pm 0.5$ & $41 \pm 2$ & $56 \pm 5$ \\
IVb HAT6 & $1585.1 \pm 0.9$ & $47 \pm 4$ & $44 \pm 5$ \\
IVa CT & $1615.1 \pm 0.7$ & $50 \pm 5$ & $75 \pm 8$ \\
IVb CT & $1584 \pm 1$ & $60 \pm 9$ & $24 \pm 8$ \\
\hline \hline
\end{tabular}

\section{DISCUSSION}

\section{A. Electron transfer in the ground state}

Intermolecular charge transfer in the electronic ground state is a well-known phenomenon for small $\pi$-conjugated molecules. ${ }^{30,31}$ But in contrast, there are only a few reports on intermolecular ground-state CT in large molecular complexes, most of them involving polymers doped with an strong electron acceptor. ${ }^{53,62}$ For discotic liquid crystalline compounds, involving D-A interaction, it is generally accepted that intermolecular charge transfer occurs in the excited state, but not in the ground state. ${ }^{27,28}$ However, we have found strong indications for a weak ground-state electron transfer in the HAT6-TNF complex. Both the observed NMR chemicalshift changes reported in the previous article ${ }^{32}$ and Raman frequency shifts are consistent with weak electron transfer from the HAT6 core to TNF, even leading to a comparable estimation for the amount of charge involved, which is about $6 \times 10^{-2}$ electron. To our knowledge, this is the first time that DFT calculations and two different experimental techniques have been used simultaneously to estimate ground-state CT effects. The strength of such combined analyses can be appreciated by considering the consistency on a more detailed level. The change in both Raman and NMR chemical shifts indicate that the electron transfer between HAT6 and TNF leads to a delocalized redistribution of the charge on TNF. For HAT6, the strongest changes in the NMR shifts and the Raman frequencies occur in the aromatic core. Furthermore, the TNF carbonyl vibration has a characteristic behavior: introduction of the electronegative $\mathrm{NO}_{2}$ substituents into the fluorenone molecule tends to increase the $\mathrm{C}=\mathrm{O}$ frequency, which can amount to $\sim 25 \mathrm{~cm}^{-1}$ within the series from aminofluorenone to TNF. ${ }^{48,63}$ The observed Raman redshift of the $\mathrm{C}=\mathrm{O}$ frequency in HAT6-TNF implies that the electron-withdrawing action of nitro substituents can be partly compensated by extra electron density donated by HAT6. This is further supported by the strong chemical shift change of the $\mathrm{C}=\mathrm{O}$ carbon observed with NMR.

The ground state $\mathrm{CT}$ in combination with the polarity of the TNF molecules concurs with the observation of a large dipole moment for HAT6-TNF in dielectric relation spectroscopy measurements. ${ }^{23}$ By using an estimated distance of $4 \AA$ between HAT6 and TNF, ${ }^{32}$ the dipole moment corresponding to a ground state transfer of $6 \times 10^{-2}$ electron is about 1.2 D. The presence of such permanent dipoles can be an important factor in facilitating charge separation of photogenerated excited CT states. ${ }^{10,13}$ It has been shown that a dipolar layer at the D-A interface can lead to a repulsive barrier separating the hole and electron residing on neighboring donor and acceptor molecules. ${ }^{14}$

\section{B. Vibrational relaxation processes in the excited states}

In Sec. III we used the resonant activity of specific molecular vibrations in the Raman spectra (Fig. 2) to characterize the different electronic excited states of HAT6-TNF. Further, the resonant Raman spectra offered an additional opportunity to identify some of the charge carrier relaxation processes 
in the excited states along with the related molecular vibrations involved in these processes. First, we consider the significantly broadened spectra obtained in the resonance mode after excitation at $325 \mathrm{~nm}$, with the $\mathrm{S}_{4}\left(A^{\prime}{ }_{1} \rightarrow E^{\prime}\right)$ electronic transition of HAT6. For both HAT6 and HAT6-TNF we found a strong enhancement of the $e^{\prime}$ symmetric quinoidal mode IV, accompanied by the appearance of a second band IVb at 1585 $\mathrm{cm}^{-1}$. Such an activity of non-totally symmetric $e^{\prime}$ modes is notable and has been observed already for the smaller building blocks of HAT6, triphenylene, and benzene. Both triphenylene and benzene are so called $(A+E) \times e$ systems, in which a doubly degenerate electronic state $E$ is vibronically coupled (pseudo-Jahn-Teller) to nondegenerate states $A$ through the degenerate $e$ vibrational modes. ${ }^{64,65}$ In the case of triphenylene, the $e^{\prime}$ mode around $1600 \mathrm{~cm}^{-1}$ has been shown to be the main channel facilitating pseudo-Jahn-Teller interactions between the lowest lying triplet states. ${ }^{24,49,64}$ In addition, it has been found that this Jahn-Teller mode provides a significant contribution to the reorganization energy of HATn molecules, which is a limiting factor for hole transport along the columnar stacks. ${ }^{61}$ For benzene it is well established that the $v_{8}\left(e_{2 \mathrm{~g}}\right)$ mode couples not only the lowest triplet states but also the lowest singlet states $B_{2 u}$ and $B_{1 u}$ with the doubly degenerate $E_{1 \mathrm{u}}$ state. ${ }^{49,65}$ Moreover, the $v_{8}$ mode splits into two channels $v_{8 \mathrm{a}}$ and $v_{8 \mathrm{~b}}$ in resonance with the strongly allowed $\left(A_{1 \mathrm{~g}} \rightarrow E_{1 \mathrm{u}}\right)$ transition when there is an $\mathrm{OH}$ or O-substituent attached to the benzene ring. ${ }^{66}$ The presence of the $\nu_{8 \mathrm{~b}}$ component implies vibronic activity ${ }^{65,66}$ since this channel gains intensity entirely from Albrecht's B-term. ${ }^{67}$ Based on the strong parentage of the HAT6 $e^{\prime}$ mode IV with the quinoidal $v_{8}$ benzene vibration, we propose that a similar vibronic mechanism is responsible for the enhancement of two components IVa and IVb, in resonance, with the $S_{4}\left(A^{\prime}{ }_{1} \rightarrow E^{\prime}\right)$ band. We have found that mode IV of HAT6 is dominated by the $v_{8}$ type of motion on the outer aromatic rings (Fig. 2), resembling the above situation of benzene with an $\mathrm{O}$ or $\mathrm{OH}$ substituent. Furthermore, the positions of the two channels IVa $\left(\sim 1616 \mathrm{~cm}^{-1}\right)$ and IVb $\left(1585 \mathrm{~cm}^{-1}\right)$ are consistent with the relative frequencies of the $v_{8 \mathrm{a}}$ and $v_{8 \mathrm{~b}}$ modes observed for the substituted benzenes, being in the range of $1589-1617 \mathrm{~cm}^{-1}$ $\left(v_{8 \mathrm{a}}\right)$ and $1560-1601 \mathrm{~cm}^{-1}\left(v_{8 \mathrm{~b}}\right)$ with a mutual separation of $15-30 \mathrm{~cm}^{-1}$. As with the lower frequency mode $\mathrm{v}_{8 \mathrm{~b}}$ in benzene, the IVb channel must gain its intensity entirely from vibronic (Albrecht's B-term) activity. The presence of this mode for excitation at $325 \mathrm{~nm}$ is therefore a strong indication that the quinoidal motion of the aromatic core is the main channel for intramolecular relaxation through vibronic coupling with lower lying electronic states. The strong broadening of the $325 \mathrm{~nm}$ spectra of HAT6 and HAT6-TNF indicates that the electron-phonon coupling processes that are involved, are fast on the Raman timescale. This is supported by the considerable Lorentzian contribution needed to obtain a good fit of the resonant spectrum, indicating a homogeneous line broadening effect.

The Raman linewidths may be used to estimate the timescale, $\tau$, of the relaxation processes within the excited state, via the energy-time uncertainty relation $\tau=\hbar / \Delta E$. $\Delta E$ being the linewidth in $\mathrm{cm}^{-1}$ and $\hbar=5.3 \times 10^{-12} \mathrm{~cm}^{-1} \mathrm{~s}^{68} \mathrm{By}$ taking the fitted FWHM of the IVb peaks (Table I) we ob- tain a timescale of 113 fs for HAT6 and 86 fs for HAT6-TNF. It seems that the excited-state relaxation processes are somewhat faster in the excited CT-complex. However, these are estimates of the minimum timescales, since other processes, like pure dephasing due to quasi-elastic events, may also contribute to the linebroadening. ${ }^{69}$ For benzene it has also been observed that the relaxation processes within the $E_{1 \mathrm{u}}$ band are fast, typically with a decay rate of $10^{14} \mathrm{~s}^{-1},{ }^{70,71}$ which is comparable to our lower bounds for internal conversion in the $E^{\prime}$ band of HAT6.

Considering the excited CT-band, the enhanced groundstate vibrational modes in the resonant Raman spectra cannot be related directly to relaxation processes, as for the $325 \mathrm{~nm}$ spectra. However, we found that the symmetric $\mathrm{NO}_{2}$ stretching vibration of TNF is the most prominent Raman active mode in the whole excited CT-band involving different electronic transitions, and that the lowest excited state involves charge transfer from the HAT6 aromatic core to TNF. From other studies it is apparent that considerable bond length and bond angle changes in the $\mathrm{C}-\mathrm{NO}_{2}$ group are involved in charge-transfer excitation of small aromatic nitro compounds. ${ }^{55,59}$ It is therefore reasonable to expect that the localized vibrations of the nitro groups play a significant role in relaxation processes within the excited CT-band. Furthermore, it appears that the relaxation processes in the CT-band are much slower than in the higher energy band. Considering the Raman linewidths, we estimate that the relaxation processes in the CT-band must be at least on the picosecond timescale, considerably slower than for the high energy HAT6 band. We tentatively argue that hot-carrier relaxation processes in the CT-band in the visible light region are relatively slow compared to the fast relaxation within the original UV absorption band of pure HAT6, which can be relevant concerning the efficient separation of charges in organic PV-devices.

\section{v. CONCLUSIONS}

We have found conclusive evidence for ground-state electron transfer in the prototypical discotic complex HAT6-TNF. The results from NMR and Raman were both consistent with weak electron transfer from the HAT6 core to TNF in the ground state, even leading to a comparable estimation for the amount of the charge involved, which is of the order of $6 \times 10^{-2}$ electron. It was shown that the excited CT-band of HAT6-TNF consists of different intermolecular electronic transitions. The lowest excited state was deduced to be predominantly a $\pi-\pi^{*}$ type of transition from the HAT6 HOMO on the aromatic core to the LUMO of TNF, the latter containing a significant contribution from the basis functions of the nitro groups. A high energy shoulder at $366 \mathrm{~nm}$ in the absorption spectrum of HAT6-TNF was observed and assigned to the strongly allowed $\mathrm{S}_{4}\left(\mathrm{~A}^{\prime}{ }_{1} \rightarrow \mathrm{E}^{\prime}\right)$ transition of pure HAT6.

We have identified a fast intramolecular relaxation process within this "hot" $\mathrm{S}_{4}$ band in both pure HAT6 and HAT6-TNF. This relaxation involves the quinoidal motion of the aromatic core, in close analogy with vibronic coupling mechanisms occurring in the building block benzene. The strong resemblance of the quinoidal relaxation process in the 
hot band of HAT6 to the case of benzene suggests that the underlying vibronic coupling mechanism is a fundamental aspect of polyaromatic hydrocarbons. In contrast, charge-carrier relaxation processes within the broad excited CT-band seem to be relatively slower than the fast internal conversion in the high energy intramolecular band of HAT6. Both the presence of permanent CT dipoles and slower relaxation processes in the CT band can be favorable concerning efficient charge separation in organic PV-devices.

\section{ACKNOWLEDGMENTS}

We gratefully acknowledge Lauren Clements and Elise Talgorn for support with the Raman and absorption measurements, and professor Robert Armstrong for critical reading. This work is part of the research program of the Foundation for Fundamental Research on Matter (FOM), which is financially supported by the Netherlands Organization for Scientific Research (NWO). This article is the result of joint research in the Delft Research Centre for Sustainable Energy and the 3TU Centre for Sustainable Energy Technologies. The Renishaw inVia Reflex Raman spectrometer and the Bruker MultiRAM Raman spectrometer, located at The University of Sydney, were purchased using a LIEF grant (LE0560680 and LE0883036) from the Australian Research Council (ARC).

${ }^{1}$ B. R. Kaafarani, Chem. Mater. 23, 378 (2011).

${ }^{2}$ W. W. Wong, C. Q. Ma, W. Pisula, C. Yan, X. Feng, D. J. Jones, K. Muellen, R. A. Janssen, P. Baeuerle, and A. B. Holmes, Chem. Mater. 22, 457 (2010).

${ }^{3}$ Y. Yamamoto, T. Fukushima, Y. Suna, N. Ishii, A. Saeki, S. Seki, S. Tagawa, M. Taniguchi, T. Kawai, and T. Aida, Science 314, 1761 (2006).

${ }^{4}$ L. Schmidt-Mende, A. Fechtenkotter, K. Mullen, E. Moons, R. H. Friend, and J. D. MacKenzie, Science 293, 1119 (2001).

${ }^{5}$ A. M. Van de Craats, J. M. Warman, A. Fechtenkotter, J. D. Brand, M. A. Harbison, and K. Mullen, Adv. Mater. 11, 1469 (1999).

${ }^{6}$ S. Sergeyev, W. Pisula, and Y. H. Geerts, Chem. Soc. Rev. 36, 1902 (2007).

${ }^{7}$ J. J. Li, Z. Q. He, H. Zhao, H. Gopee, X. F. Kong, M. Xu, X. X. An, X. P. Jing, and A. N. Cammidge, Pure Appl. Chem. 82, 1993 (2010).

${ }^{8}$ L. A. Haverkate, M. Zbiri, M. R. Johnson, B. Deme, F. M. Mulder, and G. J. Kearley, J. Phys. Chem. B 115, 13809 (2011).

${ }^{9}$ H. C. Hesse, J. Weickert, M. Al-Hussein, L. Dossel, X. L. Feng, K. Mullen, and L. Schmidt-Mende, Sol. Energy Mater. Sol. Cells 94(3), 560 (2010).

${ }^{10}$ J. L. Bredas, J. E. Norton, J. Cornil, and V. Coropceanu, Acc. Chem. Res. 42, 1691 (2009).

${ }^{11}$ C. D. Simpson, J. S. Wu, M. D. Watson, and K. Mullen, J. Mater. Chem. 14(4), 494 (2004).

${ }^{12}$ P. Samori, X. M. Yin, N. Tchebotareva, Z. H. Wang, T. Pakula, F. Jackel, M. D. Watson, A. Venturini, K. Mullen, and J. P. Rabe, J. Am. Chem. Soc. 126, 3567 (2004).

${ }^{13}$ B. Kippelen and J. L. Bredas, Energy Environ. Sci. 2, 251 (2009).

${ }^{14}$ V. I. Arkhipov, P. Heremans, and H. Bassler, Appl. Phys. Lett. 82, 4605 (2003).

${ }^{15}$ R. D. Pensack and J. B. Asbury, J. Phys. Chem. Lett. 1, 2255 (2010).

${ }^{16}$ R. D. Pensack, K. M. Banyas, and J. B. Asbury, IEEE J. Sel. Top. Quantum Electron. 16, 1776 (2010).

${ }^{17}$ E. H. A. Beckers, S. C. J. Meskers, A. P. H. J. Schenning, Z. J. Chen, F. Wurthner, P. Marsal, D. Beljonne, J. Cornil, and R. A. J. Janssen, J. Am. Chem. Soc. 128, 649 (2006).

${ }^{18}$ N. Boden, R. J. Bushby, J. Clements, and R. Luo, J. Mater. Chem. 5, 1741 (1995).

${ }^{19}$ P. S. Kumar, S. Kumar, and V. Lakshminarayanan, J. Phys. Chem. B 112, 4865 (2008).

${ }^{20}$ V. Percec, M. Glodde, T. K. Bera, Y. Miura, I. Shiyanovskaya, K. D. Singer, V. S. K. Balagurusamy, P. A. Heiney, I. Schnell, A. Rapp, H. W. Spiess, S. D. Hudson, and H. Duan, Nature (London) 417, 384 (2002).

${ }^{21}$ K. J. Donovan, K. Scott, M. Somerton, J. Preece, and M. Manickam, Chem. Phys. 322, 471 (2006).
${ }^{22}$ D. Markovitsi, S. Marguet, J. Bondkowski, and S. Kumar, J. Phys. Chem. B 105, 1299 (2001).

${ }^{23}$ O. Kruglova, E. Mencles, Z. Yildirim, M. Wubbenhorst, F. M. Mulder, J. A. Stride, S. J. Picken, and G. J. Kearley, Chemphyschem 8, 1338 (2007).

${ }^{24}$ D. Baunsgaard, N. Harrit, M. El Balsami, F. Negri, G. Orlandi, J. Frederiksen, and R. Wilbrandt, J. Phys. Chem. A 102, 10007 (1998).

${ }^{25}$ T. Kato and T. Yamabe, Chem. Phys. 325, 437 (2006).

${ }^{26}$ C. Mapelli, C. Castiglioni, G. Zerbi, and K. Mullen, Phys. Rev. B 60, 12710 (1999).

${ }^{27}$ D. Markovitsi, N. Pfeffer, F. Charra, J. M. Nunzi, H. Bengs, and H. Ringsdorf, J. Chem. Soc., Faraday Trans. 89, 37 (1993).

${ }^{28}$ T. Hirose, T. Yumoto, K. Matsumoto, S. Mitsushio, O. Kawakami, and M. Yasutake, Mol. Cryst. Liq. Cryst. 524, 68 (2010).

${ }^{29}$ D. Markovitsi, H. Bengs, and H. Ringsdorf, J. Chem. Soc., Faraday Trans. 88, 1275 (1992).

${ }^{30}$ W. G. Blann, C. A. Fyfe, J. R. Lyerla, and C. S. Yannoni, J. Am. Chem. Soc. 103, 4030 (1981).

${ }^{31}$ (a) R. S. Mulliken, J. Phys. Chem. 56, 801 (1952); (b) J. N. Murrell, J. Am. Chem. Soc. 81, 5037 (1959).

${ }^{32}$ L. A. Haverkate, M. Zbiri, M. R. Johnson, B. Deme, H. J. M. de Groot, F. Lefeber, A. Kotlewski, S. J. Picken, F. M. Mulder, and G. J. Kearley, J. Phys. Chem. B 116, 13098 (2012).

${ }^{33}$ O. Kruglova, F. M. Mulder, A. Kotlewski, S. J. Picken, S. Parker, M. R. Johnson, and G. J. Kearley, Chem. Phys. 330, 360 (2006).

${ }^{34}$ E. A. Carter, M. D. Hargreaves, N. Kononenko, I. Graham, H. G. M. Edwards, B. Swarbrick, and R. Torrence, Vib. Spectrosc. 50, 116 (2009).

${ }^{35}$ P. Hohenberg and W. Kohn, Phys. Rev. 136, B864 (1964).

${ }^{36}$ W. Kohn and L. J. Sham, Phys. Rev. 140, A1133 (1965).

${ }^{37}$ M. J. Frisch, G. W. Trucks, H. B. Schlegel, G. E. Scuseria, M. A. Rob, J. R. Cheeseman, J. A. Montgomery Jr., T. Vreven, K. N. Kudin, J. C. Burant, J. M. Millam, S. S. Iyengar, J. Tomasi, V. Barone, B. Mennucci, M. Cossi, G. Scalmani, N. Rega, G. A. Petersson, H. Nakatsuji, M. Hada, M. Ehara, K. Toyota, R. Fukuda, J. Hasegawa, M. Ishida, T. Nakajima, Y. Honda, O. Kitao, H. Nakai, M. Klene, X. Li, J. E. Knox, H. P. Hratchian, J. B. Cross, V. Bakken, C. Adamo, J. Jaramillo, R. Gomperts, R. E. Stratmann, O. Yazyev, A. J. Austin, R. Cammi, C. Pomelli, J. W. Ochterski, P. Y. Ayala, K. Morokuma, G. A. Voth, P. Salvador, J. J. Dannenberg, V. G. Zakrzewski, S. Dapprich, A. D. Daniels, M. C. Strain, O. Farkas, D. K. Malick, A. D. Rabuck, K. Raghavachari, J. B. Foresman, J. V. Ortiz, Q. Cui, A. G. Baboul, S. Clifford, J. Cioslowski, B. B. Stefanov, G. Liu, A. Liashenko, P. Piskorz, I. Komaromi, R. L. Martin, D. J. Fox, T. Keith, M. A. Al-Laham, C. Y. Peng, A. Nanayakkara, M. Challacombe, P. M. W. Gill, B. Johnson, W. Chen, M. W. Wong, C. Gonzalez, and J. A. Pople, Gaussian 03 (Gaussian, Inc., Wallingford, CT, 2003).

${ }^{38}$ J. C. Slater, The Self-Consistent Field for Molecular and Solids, Quantum Theory of Molecular and Solids (McGraw-Hill, New York, 1974), Vol. 4.

${ }^{39}$ S. H. Vosko, L. Wilk, and M. Nusair, Can. J. Phys. 58, 1200 (1980).

${ }^{40}$ M. J. Frisch, J. A. Pople, and J. S. Binkley, J. Chem. Phys. 80, 3265 (1984).

${ }^{41}$ S. Marguet, D. Markovitsi, P. Millie, H. Sigal, and S. Kumar, J. Phys. Chem. B 102, 4697 (1998).

${ }^{42}$ D. Baunsgaard, M. Larsen, N. Harrit, J. Frederiksen, R. Wilbrandt, and H. Stapelfeldt, J. Chem. Soc., Faraday Trans. 93, 1893 (1997).

${ }^{43}$ H. Chojnacki, Z. Laskowski, A. Lewanowicz, Z. Ruziewicz, and R. Wandas, Chem. Phys. Lett. 124, 478 (1986).

${ }^{44}$ L. K. Minacheva, V. S. Sergienko, S. B. Strashnova, O. V. Avramenko, O. V. Koval'chulkova, O. A. Egorova, and B. E. Zaitsev, Crystallogr. Rep. 50, 72 (2005).

${ }^{45}$ Y. S. Bulyshev, I. M. Kashirskii, and V. V. Sinitskii, Phys. Status Solidi A 82, 537 (1984).

${ }^{46}$ See supplementary material at http://dx.doi.org/10.1063/1.4856815 for the assignment of the Raman spectra, the corresponding CT shifts for HAT6TNF, and DFT calculations on TNF.

${ }^{47}$ A. Yasuda and J. Seto, Sol. Energy Mater. Sol. Cells 25, 257 (1992).

${ }^{48}$ D. Y. Paraschuk, S. G. Elizarov, A. N. Khodarev, A. N. Shchegolikhin, S. A. Arnautov, and E. M. Nechvolodova, JETP Lett. 81, 467 (2005).

${ }^{49}$ N. Nishi, K. Matsui, M. Kinoshita, and J. Higuchi, Mol. Phys. 38, 1 (1979).

${ }^{50}$ R. P. Van Duyne, T. W. Cape, M. R. Suchanski, and A. R. Siedle, J. Phys. Chem. 90, 739 (1986).

${ }^{51}$ P. J. Wagner, R. J. Truman, A. E. Puchalski, and R. Wake, J. Am. Chem. Soc. 108, 7727 (1986).

${ }^{52}$ A. SalmeronValverde, J. G. RoblesMartinez, J. GarciaSerrano, A. Zehe, R. Gomez, R. Ridaura, and M. Quintana, Cryst. Res. Technol. 32, 717 (1997).

${ }^{53}$ V. V. Bruevich, T. S. Makhmutov, S. G. Elizarov, E. M. Nechvolodova, and D. Y. Paraschuk, J. Exp. Theor. Phys. 105, 469 (2007). 
${ }^{54}$ V. V. Bruevich, T. S. Makhmutov, S. G. Elizarov, E. M. Nechvolodova, and D. Y. Paraschuk, J. Chem. Phys. 127, 104905 (2007).

${ }^{55}$ A. M. Moran and A. M. Kelley, J. Chem. Phys. 115, 912 (2001).

${ }^{56}$ C. Reichardt, R. A. Vogt, and E. Crespo-Hernandez, J. Chem. Phys. 131, 224518 (2009).

${ }^{57}$ E. Collado-Fregoso, J. S. Zugazagoitia, E. F. Plaza-Medina, and J. Peon, J. Phys. Chem. A 113, 13498 (2009).

${ }^{58}$ E. F. Plaza-Medina, W. Rodriguez-Cordoba, and J. Peon, J. Phys. Chem. A 115, 9782 (2011).

${ }^{59}$ E. D. Schmid, M. Moschallski, and W. L. Peticolas, J. Phys. Chem. 90, 2340 (1986).

${ }^{60}$ M. Zbiri, M. R. Johnson, G. J. Kearley, and F. M. Mulder, Theor. Chem. Acc. 125, 445 (2010).

${ }^{61}$ V. Lemaur, D. A. Da Silva Filho, V. Coropceanu, M. Lehmann, Y. Geerts, J. Piris, M. G. Debije, A. M. Van de Craats, K. Senthilkumar, L. D. A. Siebbeles, J. M. Warman, J. L. Bredas, and J. Cornil, J. Am. Chem. Soc. 126, 3271 (2004).
${ }^{62}$ A. Simmons and A. Natansohn, Macromolecules 24, 3651 (1991).

${ }^{63}$ G. L. Eakins, J. S. Alford, B. J. Tiegs, B. E. Breyfogle, and C. J. Stearman, J. Phys. Org. Chem. 24, 1119 (2011).

${ }^{64}$ M. Z. Zgierski, Chem. Phys. Lett. 69, 608 (1980).

${ }^{65}$ M. Z. Zgierski, M. Pawlikowski, and B. S. Hudson, J. Chem. Phys. 103, 1361 (1995).

${ }^{66}$ S. P. A. Fodor, R. A. Copeland, C. A. Grygon, and T. G. Spiro, J. Am. Chem. Soc. 111, 5509 (1989).

${ }^{67}$ A. C. Albrecht, J. Chem. Phys. 34, 1476 (1961).

${ }^{68}$ L. Bergman, D. Alexson, P. L. Murphy, R. J. Nemanich, M. Dutta, M. A. Stroscio, C. Balkas, H. Shin, and R. F. Davis, Phys. Rev. B 59, 12977 (1999).

${ }^{69}$ L. D. Ziegler, Acc. Chem. Res. 27, 1 (1994).

${ }^{70}$ Y. Achiba, K. Sato, K. Shobatake, and K. Kimura, J. Chem. Phys. 79, 5213 (1983).

${ }^{71}$ R. J. Sension, R. J. Brudzynski, and B. Hudson, J. Chem. Phys. 94, 873 (1991). 